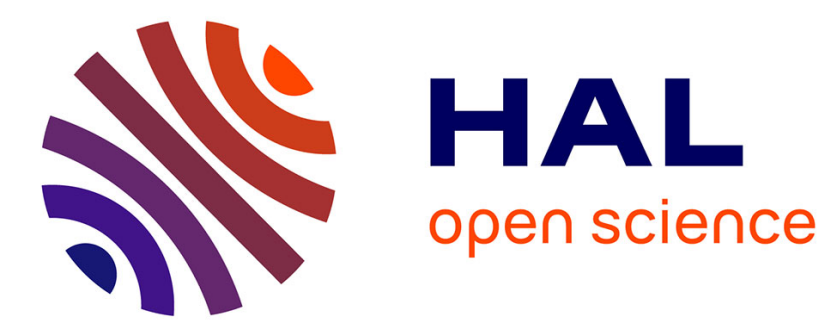

\title{
Adhesive water-in-oil nano-emulsions generated by the phase inversion temperature method
}

Nicolas Anton, Patrick Saulnier

\section{To cite this version:}

Nicolas Anton, Patrick Saulnier. Adhesive water-in-oil nano-emulsions generated by the phase inversion temperature method. Soft Matter, 2013, 9 (28), pp.6465-74. 10.1039/c3sm51064f . hal03178892

\section{HAL Id: hal-03178892 \\ https://univ-angers.hal.science/hal-03178892}

Submitted on 24 Mar 2021

HAL is a multi-disciplinary open access archive for the deposit and dissemination of scientific research documents, whether they are published or not. The documents may come from teaching and research institutions in France or abroad, or from public or private research centers.
L'archive ouverte pluridisciplinaire HAL, est destinée au dépôt et à la diffusion de documents scientifiques de niveau recherche, publiés ou non, émanant des établissements d'enseignement et de recherche français ou étrangers, des laboratoires publics ou privés. 
Cite this: DOI: $10.1039 / \mathrm{c} 3 \mathrm{sm} 51064 \mathrm{f}$

\title{
Adhesive water-in-oil nano-emulsions generated by the phase inversion temperature method
}

\begin{abstract}
Nicolas Anton ${ }^{\star a}$ and Patrick Saulnier ${ }^{b}$
This study aims at exploring and understanding the formulation of reverse (water-in-oil) nano-emulsions by the phase inversion temperature method (PIT method). Over the past decade, low-energy nanoemulsification methods have been largely approached for the formulation of direct (oil-in-water) nanoemulsions. These studies have allowed better understanding in identifying the basic concepts and principles driving the nano-droplet formation processes. In fact, the water-in-oil low-energy nanoemulsification has been much less studied and understood. Recent advances in this field have demonstrated methods and processes based on spontaneous emulsification, however there have been no studies to date describing the driving principles in the production and formulation of reverse nanoemulsions and their link with direct nano-emulsification, especially by the PIT method which still remains challenging. Herein we report for the first time a comprehensive experimental approach that explores and understands the formulation of water-in-oil nano-emulsions by the phase inversion temperature method. The experimental system for emulsion phase inversion was composed of water-paraffin oil$\mathrm{C}_{18} \mathrm{E}_{6}$ (lipophilic polyethoxylated surfactant), which was maintained in the phase inversion zone before its breaking-up by the dilution light oil (2-methylbutane), immediately generating reverse nanoemulsions. The results allowed disclosure of emulsification principles and mechanisms, in the same line as the ones acknowledged for oil-in-water nano-emulsification. The study emphasized that, similarly to direct low-energy nano-emulsification with the oil-surfactant ratio, here the water-surfactant ratio has a strong impact on the resulting nano-emulsion droplet size. Finally, these reverse nano-emulsions showed strong adhesive properties, characterized commonly with macro-emulsions but never observed with nano-emulsions, and thus leading to the formation of nano-emulsion-gels.
\end{abstract}

Received 18th April 2013

Accepted 17th May 2013

DOI: $10.1039 / \mathrm{c} 3 \mathrm{sm} 51064 \mathrm{f}$

www.rsc.org/softmatter applications can be the nano-encapsulation of drugs enhancing the bioavailability, or contrast agents for biomedical imaging applications. ${ }^{15,20-24}$ In such cases, the nature and toxicity of oil and surfactants must be adapted to the administration routes, and the droplet surface properties must be carefully controlled and functionalized.

In addition, the low-energy methods allow spontaneous formulation of nano-emulsions, which is actually a good advantage of these methods regarding industrial production. ${ }^{6,14}$ Indeed, decreasing the droplet size of an emulsion involves increasing the Gibbs free energy $\Delta G_{\mathrm{f}}$ by the interfacial area $\Delta A$ gained during processing. ${ }^{11}$ Conventional emulsification methods (rotor/stator apparatuses, colloidal mills) do not furnish enough energy to sufficiently increase $\Delta A$ in order to reach the sub-micron droplet sizes, ${ }^{7,25}$ and only specific homogenizers (high pressure homogenizers, ultrasound homogenizers) allow the generation of nano-emulsions..$^{25-27}$ Such so-called high energy processes are widely used in industries (e.g. dairy industries) but suffer from low energetic yields so much that only $0.1 \%$ of the supplied energy serves for the emulsification. ${ }^{7}$ In contrast, the possibility offered by the lowenergy methods to allow the formulation of nano-emulsions 
without supplying any energy (except for gentle mixing or heating) appears as a great alternative to high-energy processes. ${ }^{11}$ Even, it is not speculative to say that the great interest of fundamental research on these low-energy processes is closely related to the fact that they constitute a major industrial purpose. ${ }^{6}$ Some industrial fields especially the cosmetic ones have already adopted spontaneous nano-emulsification processes. ${ }^{25}$

The low-energy formulation methods strictly depend on the given specifications (e.g. using biodegradable excipients, solubilizing drugs or contrast agents at high concentrations) and can potentially affect the overall formulation processes. ${ }^{\mathbf{1 1 , 1 4 , 2 1}}$ This is why an essential point for designing new applications lies in careful understanding of the formulation processes, including the driving mechanisms and the impact of formulation and composition parameters on the physicochemical properties of the resulting nano-emulsions. Recently, we have shown that for direct nano-emulsions, ${ }^{14}$ the low-energy emulsification methods (phase inversion temperature (PIT) method and spontaneous emulsification) can be described with a universal principle. In brief, when an oil phase is mixed with a surfactant phase at the molecular level, nano-emulsification occurs with the sudden solubilization by the water phase, of the surfactant nanometric network (breaking-up the oil-surfactant phase).${ }^{14}$ As a result, the oil phase undergoes spinodal demixing forming the nano-emulsion droplets, immediately stabilized by surfactants. The success of the process is related to the control and understanding of the surfactant's affinity for water and oil, generally done by temperature. In the case of the PIT method, $\mathrm{T}>$ PIT signifies that surfactants are lipophilic, and a rapid cooling of the system suddenly makes them hydrophilic, thus inducing water penetration in oil. To summarize, as regards direct or oil-in-water nano-emulsions, the underlying principles for the spontaneous formulation processes are accurately described and understood. ${ }^{\mathbf{1 4 , 1 7}}$ Actually, a large majority of examples only concerns the studies related to the low-energy nano-emulsification processes for the direct nano-emulsions. This simply corresponds to the needs and applications of current nanotechnologies, which are largely devoted to nanoparticulate systems dispersed in the aqueous phase. For example, designing nanomedicines also includes the specifications of administration routes (parenteral, ocular, oral, topical, etc.), for which the continuous phase must be aqueous.

On the other hand, for this very reason, the low-energy methods aiming at the formulation of reverse or water-in-oil nano-emulsions have been much less studied. The new challenges faced by today's biomedical research aim at the development of a new generation of nanocarriers that are able to encapsulate hydrophilic bioactive smart materials (therapeutic peptides, oligonucleotides...). One solution could possibly be the encapsulation of hydrophilic species in the aqueous core of reverse nano-emulsion droplets, followed by the fabrication of nano-capsules with interfacial polymerization or nanoprecipitation. ${ }^{\mathbf{1 3 2 8 , 2 9}}$ The result is the formulation of aqueouscore nanocarriers dispersed in water. Such innovative applications illustrate the emerging interest that reverse nanoemulsions arouse. However, presently there are only a handful of recent publications ${ }^{\mathbf{3 0 - 3 4}}$ that are based on reverse nanoemulsions generated by spontaneous emulsification. These results have emphasized that the conventional methods and rules for formulating direct nano-emulsions (PIT method, spontaneous nano-emulsification) are not directly transposable and applicable to the formulation of inverse nano-emulsions. Even, the applicability of the concepts driving the low-energy nano-emulsification, to water-in-oil systems, is still not well understood. The objective of the present study is to gain new insights into the principles related to the nano-emulsification of reverse nano-emulsions by low-energy methods, notably by the PIT method. The importance of this work lies in the fact that to date the PIT process for the formulation of reverse nanoemulsions has never been carefully studied, characterized, or understood. Precisely we will focus on the feasibility of processes and the impacts of the formulation and composition parameters on the formulation mechanisms, in order to draw a parallel relationship between the concepts driving the direct and reverse low-energy nano-emulsification.

Finally, we have demonstrated that these reverse nanoemulsions, in contrast to the direct ones, can present particular adhesive properties inducing their gelation, a phenomenon that has never been reported with nano-emulsion droplets, which we have characterized and understood by various methods.

\section{Adhesive nano-emulsions: theoretical background}

In order to fully understand the behavior of adhesive nanoemulsions once they are generated, it is necessary to present an overview of the physical concept driving the follow-up and characterization of their aggregating behavior. This is what we will do in this section. First of all, it has to be pointed out that the main feature of nano-emulsions lies in their great stability in the form of suspended droplet suspension. The main reason for this superior stability is due to the very small size of droplets that prevents gravitational effects like sedimentation or creaming. Nano-emulsion droplets can thus be related to Brownian particles, which reduce the potential flocculation and coalescence. The other point lies in the fact that flocculation is naturally prevented by steric stabilization for physical reasons (not detailed here), mainly due to the sub-micrometric size of droplets. As a result, nano-emulsion droplets have a homogeneous and stable repartition in the whole sample volume. Actually, nano-emulsions are kinetically stable (for months) but remain thermodynamically unstable. Their destabilization is mainly due to the diffusion of the dispersed phase through the continuous one, so-called Ostwald ripening.

On the other hand, in the case of reverse nano-emulsions and compared to direct ones, the parameters that confer them adhesive properties are different. Adhesive emulsion droplets exhibit a strong attraction for each other, giving rise to flocculated aggregates (not necessarily followed by coalescence). This comes when the attractive intermolecular interactions override the repulsive ones, e.g. for direct emulsions when the electrostatic repulsion is screened upon an increase of the ionic strength of the bulk. ${ }^{25,35}$ In the case of reverse emulsion, owing 
to the fact that the surfaces are not charged, the repulsive component can be negligible, and the attractive properties of the droplets are only linked to the favorable or unfavorable surfactant-oil interactions. ${ }^{36}$ To summarize, (i) if the aliphatic part of the surfactant has a good affinity with oil, two drops overlapping will strongly repulse each other (steric repulsion) and in contrast (ii) surfactants poorly soluble in oil will result in a very strong attraction between droplets. Even, this attraction can be strong enough to destabilize nano-emulsions up to the formation of a macro-aggregate only made with flocculated nano-droplets. The present study is an illustration of this phenomenon.

We will see that adhesive properties of nano-emulsions can be analyzed and followed-up according to the colloid aggregation model based on von Smoluchowski's approach, ${ }^{37}$ for Brownian particles having strong attractive properties. Numerous advances concerning the understanding of colloidal aggregation have been made in the past few decades. ${ }^{38-40}$ The structure of aggregates was shown to be scale invariant ${ }^{\mathbf{4 1}}$ and so, assumed to present a fractal geometry. Even, aggregation processes have been recognized as universal, and the clusters formed thermodynamically stable. Such a universal characteristic of the aggregation phenomenon provides two most prominent features: reaction kinetics and cluster morphology. Aggregation kinetics is described by von Smoluchowski's equation ${ }^{37}$ and the cluster structure characterized by the fractal dimension, $d_{\mathrm{f}}{ }^{\mathbf{4 2 , 4 3}}$ also called Hausdorff's dimension. The fractal dimension can be considered as being the aggregate density, since randomly shaped obtained aggregates are usually described through the fractal scaling relationship, ${ }^{\mathbf{4 4 - 4 6}}$

$$
\mathscr{M}=k_{\mathrm{f}}\left(\frac{R_{\mathrm{g}}}{R_{0}}\right)^{d_{\mathrm{f}}}
$$

where $\mathscr{M}$ is the dimensionless mass of an aggregate, $R_{\mathrm{g}}$ the radius of gyration, $R_{0}$ the primary particle radius and $k_{\mathrm{f}}$ the fractal prefactor.

In the case of colloids the aggregation kinetics of particles is governed by their approach and sticking together under the influence of Brownian motion, and is usually treated within the scaling approach of the von Smoluchowski's eqn (2). ${ }^{38,43,47}$ Furthermore, aggregation is typically described with a distribution of cluster masses $N_{M}(t)$ (or the number of clusters having the mass $M$ ) and the rate equation (Smoluchowski's equation) that describes the temporal variation of this cluster's mass distribution:

$$
\frac{\mathrm{d} N_{M}(t)}{\mathrm{d} t}=\frac{1}{2} \sum_{i+j=M} K_{i j} N_{i}(t) N_{j}(t)-\sum_{i} K_{i M} N_{M}(t) N_{i}(t)
$$

where the first term accounts for the formation of aggregates of mass $M$ in subunit clusters of mass $i$ and $j$, and the second term for the disappearance of such a cluster due to its own aggregation. The rate at which $i$-mass clusters join to $j$-mass ones is configured by the kernel $K_{i j}$ which contains all physical, chemical, and statistical information. ${ }^{48}$ This equation has been often solved numerically in order to follow full cluster mass evolution over time. ${ }^{45,46,49,50}$ Thereby, it is possible to obtain both the fundamental characteristics of an aggregation process and the regime of universal aggregation.

Two universal regimes have been found for colloidal aggregation: "diffusion limited cluster aggregation" (DLCA), also called the Witten and Sander model, ${ }^{51}$ and "reaction limited cluster aggregation" (RLCA). ${ }^{4752-57}$ The sticking probability $p$ is equal to one for DLCA and lower than one for RLCA, the probability being that one collision between two droplets gives rise to doublet formation. The RLCA regime occurs when repulsive interaction exists between colloids, though not strong enough to generate reversible flocculation. Thus, the RLCA regime leads to more uniform, compact aggregates. Reorganization within the aggregate following formation is also discussed in the RLCA regime. ${ }^{53,58}$ Static light scattering ${ }^{53,54}$ has been used to characterize cluster morphology and kinetic regimes with the fractal dimension $d_{\mathrm{f}}$ determination. In fact, as reported in ref. 59, an approximate threshold of $d_{\mathrm{f}} \sim 2$ for a three-dimensional system separates the two aggregation regimes $\left(d_{\mathrm{f}}>2\right.$ and $d_{\mathrm{f}}<2$, respectively for RLCA and DLCA regimes). The scattered intensity by a cluster of mass $M$ can be written as being proportional to the structure factor $S\left(q R_{\mathrm{g}}\right)$ and the form factor $F(q)$ as

$$
I_{\mathrm{M}}(q) \sim M^{2} S\left(q R_{\mathrm{g}}\right) F^{2}(q)
$$

function of the wave vector $q$

$$
q=\frac{4 \pi n}{\lambda_{0}} \sin \left(\frac{\theta}{2}\right)
$$

where $n$ is the refractive index of the continuous phase, $\lambda_{0}$ is the wavelength of incident light, and $\theta$ the scatter angle. We will assume that initial particle sizes are small enough to suppose $F(q)=1$, for all experimental values of $q$. In fact, $F(q)$ is considered to be equal to 1 whether $\lambda_{0} / 20$ is higher than the particle size, and this condition was assumed regarding the initial particle size, which will be presented in the following sections. The scale invariance of fractal morphology means that the product $q R_{\mathrm{g}}$ is the only variable of the structure factor $S\left(q R_{\mathrm{g}}\right)$, and its asymptotic behavior for $q R_{\mathrm{g}} \gg 1$ is assumed to be $\mathrm{b}^{54}$ $S\left(q R_{\mathrm{g}}\right) \sim\left(q R_{\mathrm{g}}\right)^{-d}{ }_{f} \sim M^{-1} q^{-d}{ }_{f}\left(\right.$ with eqn (1)), thus $I_{M}(q) \sim M q^{-d}$. In this case, the fractal correlations of the cluster structure are resolved. For $q R_{\mathrm{g}} \ll 1$, the structure factor is equal to one, i.e. the scattering is totally coherent, and the clusters are considered as punctual objects and $I_{M}(q) \sim M^{2}$. In the present work, the fractal validity domain is considered as the $q R_{\mathrm{g}} \gg 1$ condition, where the cluster size becomes sufficiently large, hence $q$ satisfies the condition:

$$
R_{\mathrm{g}}{ }^{-1} \ll q \ll R_{0}^{-1}
$$

Thus, globally measured scatter intensity is the weighted sum over the cluster mass distribution:

$$
I(q)=\sum_{M} N_{M}(t) I_{M}(q) \sim \sum_{M} N_{M}(t) M q^{-d_{\mathrm{f}}}
$$

that finally leads to the following relationship that is useful to determine the fractal dimension: 


$$
I(q) \sim q_{f}^{-d}
$$

Outside the validity conditions, eqn (5), only a Guinier law $\left(q \leq{R_{\mathrm{g}}}^{-1}\right)$ or elementary particle diffusion $\left(R_{0}{ }^{-1} \leq q\right)$ can be observed, i.e. no linear relationship between $\log (I)$ and $\log (q)$ could result.

\section{Materials and methods}

\subsection{Materials}

A technical grade lipophilic polyethoxylated surfactant $\mathrm{C}_{18} \mathrm{E}_{6}$ was kindly furnished by Stearinerie-Dubois (Boulogne, France). It is a typical commercial product with a Poisson-like ethylene oxide (EO) distribution around six. Light mineral oil was purchased from Cooper (Melun, France), and it corresponds to a standardized denomination to refer to a mixture of saturated hydrocarbons obtained from petroleum. Ultrapure water was obtained using a MilliQ ${ }^{\circledR}$ filtration system (Millipore, SaintQuentin-en-Yvelines, France) and sodium chloride from Prolabo (Fontenay-sous-Bois, France). Finally, 2-methylbutane was obtained from Riedel-de-Haën (Germany).

\subsection{Methods}

3.2.1 Nano-emulsion formulation. Nano-emulsions were prepared according to the phase inversion temperature method. This conventional method was adapted to generate water-in-oil nano-emulsions. The formulation was composed of three steps: (i) the formulation of a macro-emulsion composed of paraffin oil, water plus $\mathrm{NaCl}(0.51 \mathrm{M})$, and nonionic surfactant. In our previous report ${ }^{\mathbf{1 0}}$ we have shown a link with the PIT location and electrolyte concentration. Actually, we choose here to introduce $\mathrm{NaCl}$ into the water phase for two reasons (i) to increase the electrical conductivity and enable following the emulsion phase inversion and (ii) because it increases the lipophilicity of surfactants favoring improvement in the water-inoil nano-emulsion formation process. In order to simplify the study, $\mathrm{NaCl}$ concentration was fixed to a representative value, $0.51 \mathrm{M}$. The composition is fixed by the water-to-oil weight ratio, WOR $(=100 \times($ water mass $) /($ water + oil masses $))$, and the surfactant amount (in wt\%). The macro-emulsion is gently magnetically stirred $(\sim 500 \mathrm{rpm})$, and its temperature is controlled in a thermostatted water bath varying from $25{ }^{\circ} \mathrm{C}$ to $60{ }^{\circ} \mathrm{C}$. (ii) Once the macro-emulsion is homogenized, the system is brought to and maintained in the phase inversion zone (PIZ), precisely at the PIT (determined as described below), and still under moderate stirring. The milky aspect of the macroemulsion changes into the bluish and transparent one of microemulsions. (iii) Finally, the nano-emulsification is achieved by the dilution of the microemulsion with 2-methylbutane at room temperature, with a volume of 2-methylbutane at least twice the microemulsion one, and still under gentle stirring. Water-in-oil nano-emulsions are immediately generated. Note that now the bulk continuous phase is a mixture of paraffin oil and 2-methylbutane.

3.2.2 Phase inversion temperature and formulationcomposition maps. The phase inversion temperature is determined by following-up the electrical conductivity of the emulsion as a function of the temperature. A conductimeter (Cond 330i/SET, WTW, Germany) was used in nonlinear temperature compensation mode, according to EN 27 888. High steady conductivity (i.e. $\sim \mathrm{mS} \mathrm{cm}^{-1}$ ) indicates that the continuous phase is water, whereas conductivity about $10 \mu \mathrm{S} \mathrm{cm}^{-1}$ or lower (null in the illustrated scales below) means that the continuous phase is oil. Each experiment was performed three times. Conductivity experiments allow the emulsion phase inversion to be revealed, and therefore identification of the domains in which the surfactants are soluble in water, in oil, or in both. In addition, they allow disclosure of regions of the conductivity peak corresponding to particular microemulsion structures. Accordingly, the formulation-composition maps are built by reporting these domains in diagrams, as a function of the temperature and the WOR. The linear parts of the electrical conductivity curves were extrapolated giving the transition temperatures between the different domains from their intersections (as illustrated in previous works ${ }^{\mathbf{1 0}}$ ). The limits of the peak domains were defined with the increase of the conductivity around the peak.

3.2.3 Static and dynamic light scattering. Static light scattering (SLS) and dynamic light scattering (DLS) were carried out using a Malvern 4700 Autosizer ${ }^{\circledR}$ (Malvern, Orsay, France) at $20{ }^{\circ} \mathrm{C}$, with a scatter angle varying from $10^{\circ}$ to $145^{\circ}$. The argon ion, air-cooled laser used operates at $488 \mathrm{~nm}$, and the incident intensity was monitored. All measurements were performed under standard conditions in terms of sample dilution, and at constant temperature, ensuring the reproducibility of the experimental conditions. After fabrication, a sample of nanoemulsion around $1 \mathrm{~mL}$ was placed in the apparatus. DLS data were analyzed using a cumulant-based method. PDI is a measure of the broadness of a size distribution derived from the cumulant analysis of DLS data according to ISO 13321:1996; for a single Gaussian population with standard deviation, $\sigma$, and mean size, $x_{\mathrm{PCS}}$, thus PDI $=\sigma^{2} / x_{\mathrm{PCS}}{ }^{2}$ is the relative variance of the distribution. In other words, it shows the quality of the dispersion. Values $\leq 0.1$ reflect a very good monodispersity and quality of the nanoparticulate suspensions. Measurements were performed three times for each point. DLS measurement requires a precise value of the refractive index and the viscosity of the continuous phase. The refractive index of this medium was obtained using a Reichert AR700 temperature controlled, automatic refractometer (Reichert, Inc., USA). Viscosity was measured using a capillary viscosimeter Schott Gerate AVS400 device (Schott Gerate, Germany), the tube constant was $K=$ $9.636 \times 10^{-6} \mathrm{~m}^{2} \mathrm{~s}^{-2}$. In SLS mode, we followed the values of the mean intensity $I$ as a function of the wave vector $q$ (as discussed above). On the other hand, DLS measurements were carried out at a fixed wave vector $q$, for $\theta=90^{\circ}$, in oil (mixture of 2-methylbutane plus light mineral oil).

3.2.4 Interfacial tension measurements. Interfacial tension was measured with a drop tensiometer, Tracker (Teclis, Longessaigne, France). Once the temperature of the thermostatted cell is stabilized, a rising drop of paraffin oil containing nonionic surfactant is formed and maintained at a constant volume. Drop volumes were chosen to reach a Bond number around $0.2-0.25$. The droplet shape is recorded in real time with 
a video camera. As a result, the Laplacian shape of the drop gives its interfacial area and surface tension. After the drop formation, the value of the surface tension was followed over time, up to stabilization. Then the value of equilibrium interfacial tension at temperature $T, \gamma_{\mathrm{eq}}^{T}$, was collected. Finally, equilibrium interfacial pressure was accessed with the one of interfacial tension without surfactant $\gamma_{0}^{T}$, as $\Pi_{\text {eq }}^{T}=\gamma_{0}^{T}-\gamma_{\text {eq }}^{T}$. Different surfactant concentrations were studied, 0.25, 2.5, 10 and $50 \mu \mathrm{g} \mathrm{mL} \mathrm{mL}^{-1}$, and the temperature was varied over the emulsion phase inversion domain, from $25^{\circ} \mathrm{C}$ to $60{ }^{\circ} \mathrm{C}$.

\section{Results and discussion}

\subsection{Formulation}

In the studies related to emulsion phase inversion, determination of PIT, and the related nano-emulsification process, the first important experimental result lies in the characterization of the phase inversion through establishing the so-called formulation-composition maps. These diagrams are summarized in Fig. 1(a), for four representative surfactant amounts, 5, 7.5, 10 and $12.5 \mathrm{wt} \%$. As expected, the location of the phase (a)
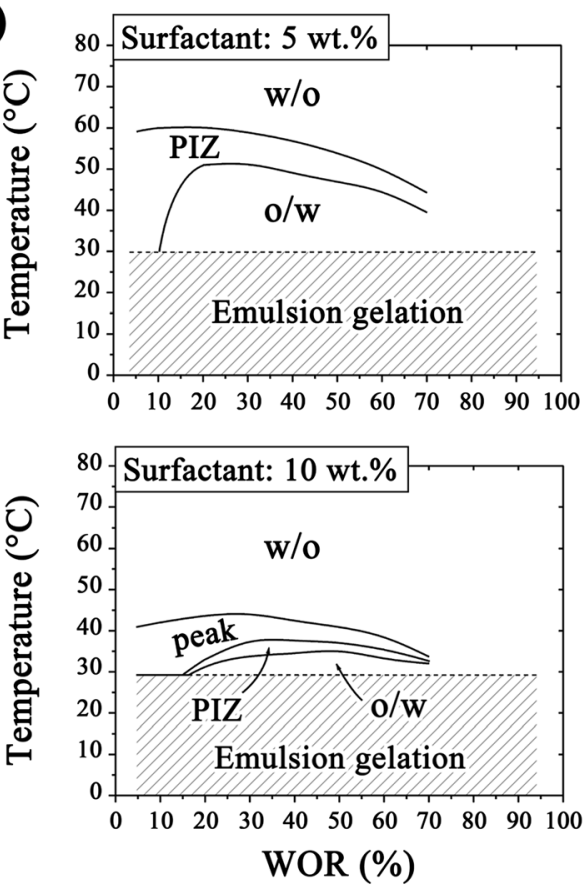
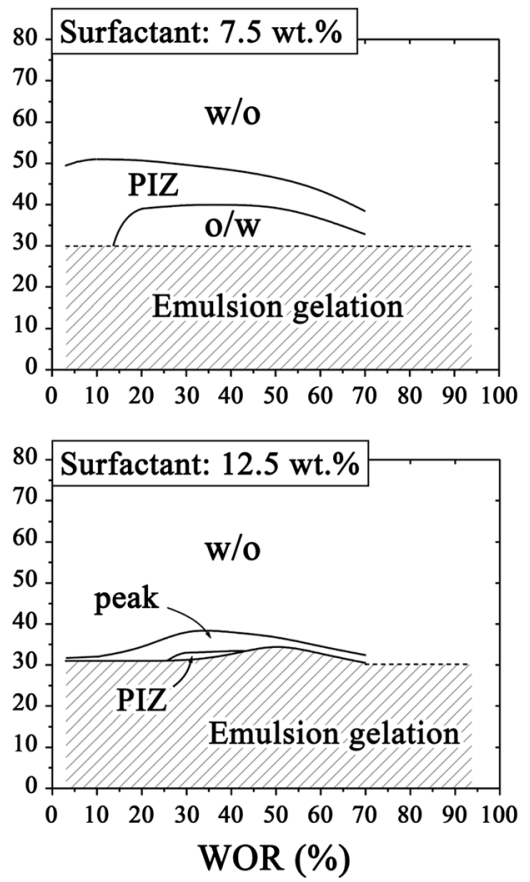

(b)
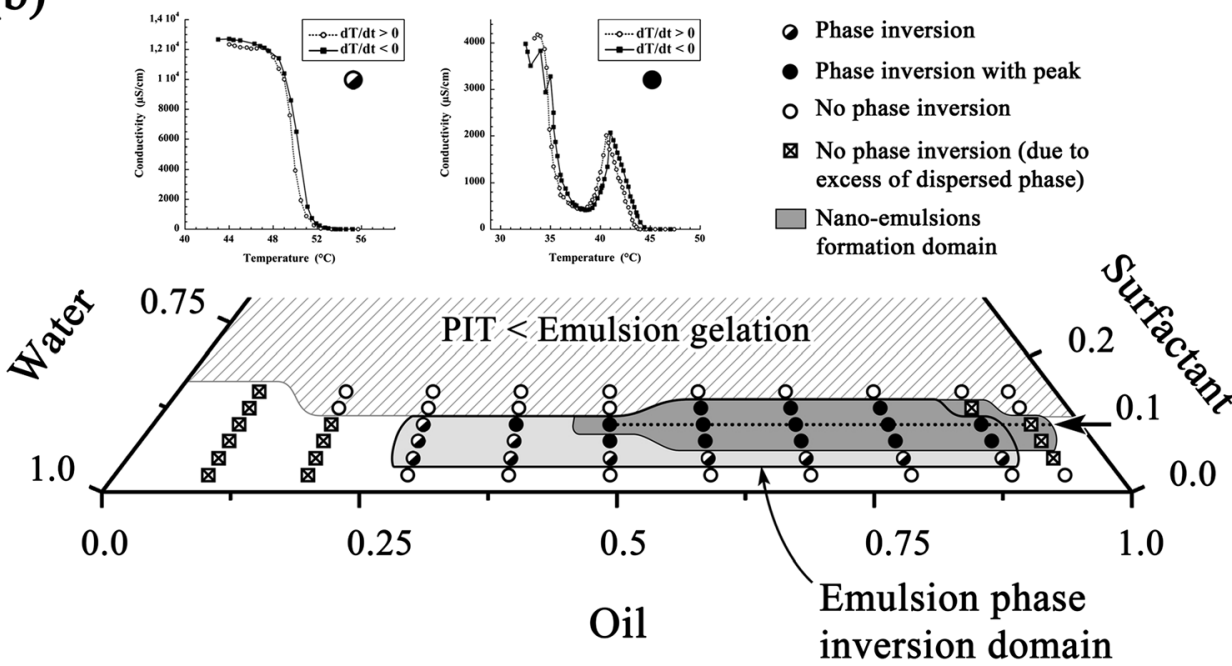

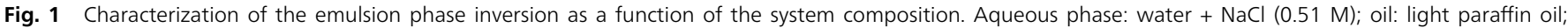

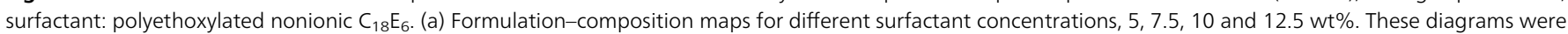

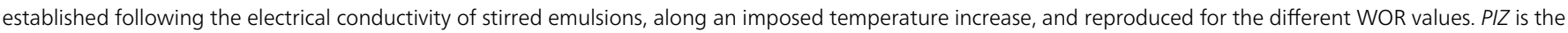

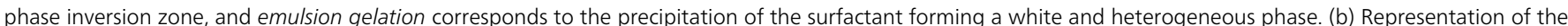

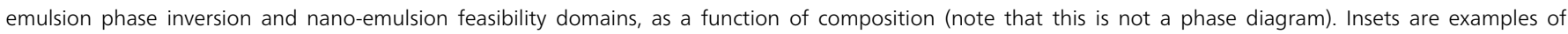
conductivity experiments, with or without peaks. 
inversion is influenced by the amount of surfactant, which shifts towards lower temperatures when increased. This means that, at a given temperature, the interface becomes more lipophilic with a rise of surfactant concentration.

Actually, it has to be kept in mind that these experiments were performed under dynamic conditions, and the studied emulsions were under continuous stirring when the conductivity measurements were performed. Thus, as occurs in the classical emulsification processes, ${ }^{25}$ the droplets constantly undergo break-up and coalescence. The emulsion droplet size and the water-oil interface properties depend on the amount and kinetics of surfactants that adsorbed onto the newly formed droplets after breaking-up and before coalescence. This explains the observed shifts of the inversion zones, i.e. increasing the surfactant concentration results in a higher amount of surfactants adsorbed at the interface, and finally modifying the interface properties. ${ }^{10}$ The lowering of the phase inversion temperature confirms the lipophilic interfacial properties of the $\mathrm{C}_{18} \mathrm{E}_{6}$ nonionic surfactant used. It is to be noted that the decrease of the emulsion phase inversion zone has been discussed in the literature, ${ }^{6}$ and another explanation comes from the Poisson-like distribution of the molecular weight of the EO groups of technical grade surfactants. This induces an interfacial segregation of the shorter molecules (i.e. more lipophilic) as the concentration is increased, thus resulting in lowering of the PIT. Nevertheless, the fact that emulsions invert indicates that, below the PIT, the surfactants still become hydrophilic and are solubilized by the water phase. This change in affinities towards oil and water is indirectly observed and confirmed by following the interfacial pressure $\Pi$ as a function of the temperature, with model dilute systems. Lipophilic $\mathrm{C}_{18} \mathrm{E}_{6}$ surfactants are solubilized in paraffin oil, forming rising drops in water. The equilibrium interfacial pressure $\Pi_{\mathrm{eq}}$ is recorded for a given temperature, varying from $25^{\circ} \mathrm{C}$ to $60{ }^{\circ} \mathrm{C}$. The results are reported in Fig. 2, $\Pi_{\text {eq }}$ appears significantly sensitive to temperature, decreasing when the temperature increases. Of course these model interfaces are very diluted as compared to the actual emulsification conditions, but they allow studying the impact of the temperature on the surfactant behavior. Interfacial pressure shows a linear increase as the temperature decreases, around $\Delta \Pi_{\mathrm{eq}}=13 \mathrm{mN} \mathrm{m}^{-1}$ whatever may be the concentration, signifying that either more surfactants are adsorbed at the interface, or their affinity for water (and interfacial activity) is improved. Finally this confirms that such lipophilic surfactants can increase their affinity towards water under specific temperature conditions. In addition, this experimental approach emphasizes that the phase inversion temperature is not linked to a particular behavior of the thermosensitive surfactant at a given temperature, but rather only to its change in affinities for water and oil as a function of temperature, and thus to their migration from one to another phase, still consistent with the low-energy mechanisms we have proposed for direct nano-emulsification. ${ }^{\mathbf{1 4}}$

Owing to the above discussion, the conductivity peak reflects an increased interfacial concentration leading to the formation of specific bicontinuous phases (liquid crystalline phases, sponge-like microemulsions) forming aqueous (conductive)

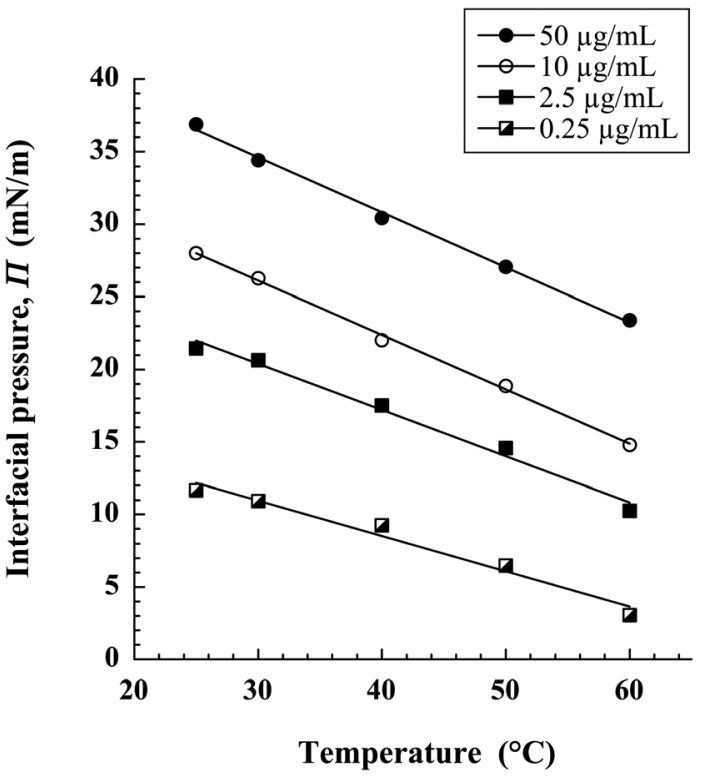

Fig. 2 Equilibrium surface pressure at the interface of a rising oil drop in water, as a function of the surfactant concentration and temperature. Aqueous phase: water $+\mathrm{NaCl}(0.51 \mathrm{M})$; oil: light paraffin oil; surfactant: polyethoxylated nonionic $\mathrm{C}_{18} \mathrm{E}_{6}$.

channels. The solubility of surfactants still changes, being shared between oil and water in the peak region or PIZ, and that is also true for the $\mathbf{1 2 . 5} \mathrm{wt} \%$ system even if no phase inversion occurs. To sum up, the emulsion inversion domain is limited (i) at high WOR values, by a region where an excess of the dispersed phase prevents emulsion phase inversion, and (ii) at $T<30{ }^{\circ} \mathrm{C}$ by the emulsion gelation (due to the surfactant precipitation).

The qualitative results obtained by formulation-composition maps are reported in a ternary diagram Fig. 1(b), and compared with the regions where the nano-emulsification is successful. It should be noted that the phase inversion is not always associated with the nano-emulsification, which is also the case for the direct nano-emulsification. ${ }^{9}$ However, it roughly corresponds to the region in which the surfactants exhibit better interfacial activity, characterized by the presence of conductivity peaks. ${ }^{12}$ Likewise it still corresponds to the part of the diagram for which WOR $<50 \%$, with the lower amount of water. The upper limit of the phase inversion zone and the nano-emulsion formation domain is due to a further decrease of the PIT below the emulsion gelation (in the same line as the PIT decrease observed when the surfactant amount is increased). As well, at very low WOR, the phase inversion does not occur because of an excessive amount of oil, and the aqueous phase is not able to become the continuous one, even if it solubilizes the majority of surfactants (due to temperature effects). The globules can present multiple emulsion structures. This is verified since reverse nano-emulsions can be generated also in this region (squares in the oil corner, in Fig. 1(b)). This case of PIT nano-emulsification in regions of excess of the dispersed phase can be, as well, found in the case of oil-in-water nano-emulsions, as we previously reported. ${ }^{60}$ This only 
corresponds to a limit case of extreme WOR, but it is still coherent with the nano-emulsification breaking-up mechanisms. Finally, it is possible to identify regions where the emulsion inversion shows conductivity peaks, but without generation of nano-emulsions (for higher WOR values): the droplet size of the forming emulsions cannot reach nanometric scales because of the high water amount.

Now, in order to understand what could be the emulsification mechanisms, and if there is a possible analogy between direct and inverse nano-emulsification, let us focus on the ones driving the low-energy methods, generating oil-in-water nanoemulsions. We have previously demonstrated ${ }^{\mathbf{1 4}}$ that it can be likely linked (for oil-in-water nano-emulsions) with the penetration of water in the surfactant-oil homogeneous phase, when surfactants undergo a sudden change in solubility towards the aqueous phase. This was confirmed by the size decrease of the droplets when the oil amount is reduced, ${ }^{\mathbf{1 4 , 2 1 , 2 4}}$ at a constant surfactant concentration. We also discussed the universality of such principle since it is observed irrespective of the low-energy emulsification way (PIT method or spontaneous emulsification). ${ }^{14}$ In the present study, aiming at the formulation of waterin-oil nano-emulsions, the results shown in Fig. 1(b) actually display a comparable trend. The efficiency of the formulation process (i.e. low droplet size and PDI) is increased in the region with less water and more surfactants, exactly the symmetrical rule for direct nano-emulsification (less oil and more surfactants). Accordingly, emulsification is induced by the spinodal decomposition of water in oil bulk, after the dilution oil (2methylbutane) penetrated the water-surfactant homogeneous phase by instantaneously solubilizing the lipophilic amphiphiles. Furthermore, we confirmed this hypothesis as shown in Fig. 3, where the nano-droplet sizes (immediately after formation) are plotted against the WOR at a constant surfactant

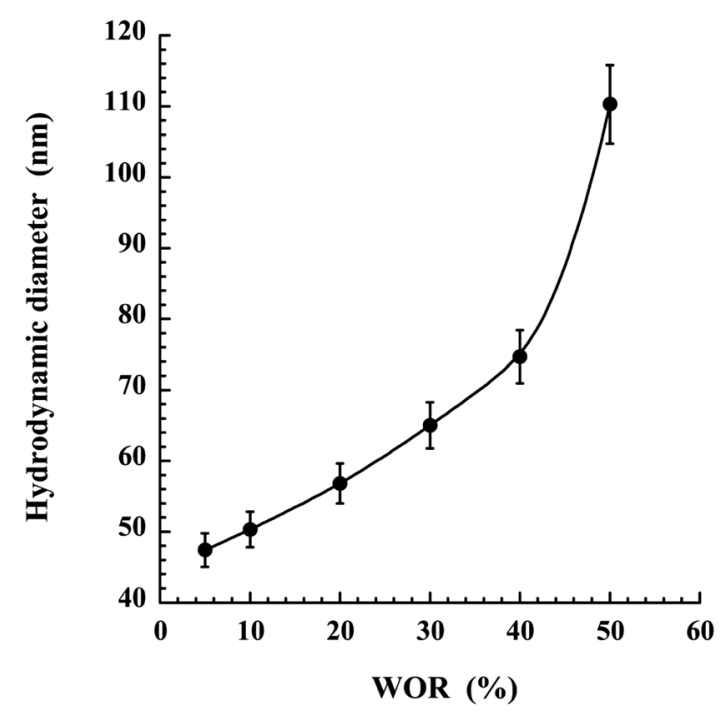

Fig. 3 Water-in-oil nano-emulsions formulated by the PIT method. Droplet size is reported as a function of the water-to-oil weight ratio (WOR) of the primary emulsion that undergoes a phase inversion, and at constant surfactant amount = 10 wt\%. Aqueous phase: water $+\mathrm{NaCl}(0.51 \mathrm{M})$; oil: light paraffin oil; surfactant: polyethoxylated nonionic $\mathrm{C}_{18} \mathrm{E}_{6}$. The composition of these emulsions before the oil dilution step corresponds to the dashed line indicated by an arrow in Fig. 1. amount $(=10 \mathrm{wt} \%$, corresponding to the dashed line indicated by the arrow in Fig. 1(b)). By analogy and consistently with direct nano-emulsification, the size appears to be clearly influenced by the water-surfactant ratio. The dilution oil, 2-methylbutane, having viscosity twice lower than water, instantaneously penetrates the surfactant network, as it is highly able to solubilize it at the PIT. This high fluidity of the dilution oil is a prerequisite for such reverse nano-emulsification, finally consistent with the supposed mechanism. To summarize, these unprecedented results show that the phase inversion temperature method not only can allow generation of water-in-oil nano-emulsions, but also according to the universal mechanisms described for the oil-in-water nano-emulsification. As a last remark, it is to be noted that the low molecular weight and low viscosity of the dilution oil are fundamental points for the success of the nano-emulsification process. Indeed, if the microemulsion is diluted by the same "mineral oil" (than the one constituting the microemulsion), the formulation of nanoemulsion does not work. Low viscosity dilution oil is absolutely necessary for the formulation of inverse nano-emulsions with the process described here.

\subsection{Stability}

Once formed, the hydrodynamic diameter of the nano-emulsion droplets accessed by DLS undergoes a rapid growth over time, as reported in Fig. 4(a) for WOR =10, and Fig. 4(b) for WOR = 40. At equilibrium, the nano-emulsion forms a bluish and translucent gel precipitate as reported in the picture in Fig. 4, indicating that any phase separation occurred, but rather, that the nano-droplets seem to be aggregated. In the first part, the nano-emulsions appeared very monodispersed with a PDI $<0.1$. Then, the droplet size gradually increases, along with the PDI, up to DLS measurements are not valid (PDI > 0.5), actually corresponding to the cloudy aspect of the suspension just before the macro-aggregate precipitates. At this time, an aggregating behavior of nano-droplets has been suspected, and definitively confirmed by the SLS experiments, reported in Fig. 5. Indeed, the mean intensity $I$ expressed as a function of the wave vector $q$ satisfies eqn (7) with a good correlation. This result confirms that a colloidal aggregation effectively occurs between the nano-emulsion droplets, and gives rise to fractal objects characterized by their fractal dimension $d_{\mathrm{f}}$ (as detailed in the theoretical part above Section 2).

As presented above in Section 2, such aggregating behavior is very uncommon with nano-emulsions, since most studies are focused on oil-in-water nano-emulsions, and in water the repulsive contributions of the interdroplet forces are much higher than in oil. Moreover, we saw that unfavorable mixing between surfactant and bulk oil can result in strong attractive forces. This is precisely what is supposed to occur in the present system, proved by their aggregating behavior, and simply explained by the temperature effect. Indeed, the formulationcomposition maps (Fig. 1(a)) showed that the phase inversions occur above $30{ }^{\circ} \mathrm{C}$, meaning that after fabrication when they are at storage temperature (room temperature around $25{ }^{\circ} \mathrm{C}$ ), the droplet stabilizing surfactant layer becomes hydrophilic and 

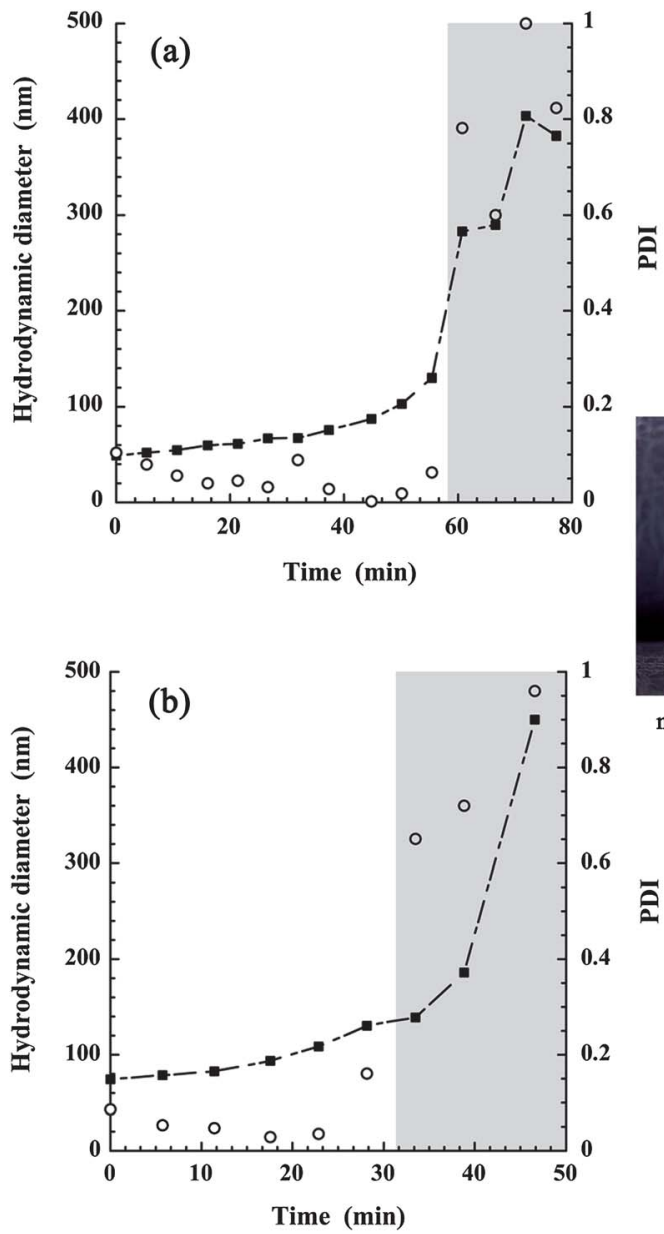

Fig. 4 Dynamic light scattering characterization of the water-in-oil nano-emulsions over time. Hydrodynamic experiments and polydispersity indices are reported as a function of the time after the nano-emulsion generation (after oil dilution). The gray part indicates regions in which the PDI is too high to consider the measurements valid. (a) $W O R=10 \%$ and (b) WOR $=40 \%$. Photograph shows the visual aspect of the nano-emulsion, after the colloidal aggregation of nanodroplets is achieved, forming a bluish and translucent nano-emulsion-gel.

loses its solvation in the oil bulk phase. This effect can even more be increased by the fact that we are below the surfactant precipitation temperature, where they are insoluble in both water and oil. Such a system exactly illustrates the case of unfavorable mixing known for macro-emulsion, ${ }^{36}$ and has never been observed with nano-emulsions. They accordingly form nano-aggregates.

Another interesting result concerns the value of the fractal dimension of the forming aggregates. When the scale invariance geometry of the clusters begins to be detectable in the suspension (i.e. eqn (7) is satisfied), the values of $d_{\mathrm{f}}$ appear $\sim 2$, close to the RLCA regime. It follows that the nano-emulsion droplets are loosely bound and are able to rearrange and form compact clusters. However, $d_{\mathrm{f}}$ decreases along the aggregation process. This is not due to the increase of the cluster size since the fractal dimension is independent of the scale, but rather due to a gradual decrease of the compactness of aggregates. This phenomenon is likely driven by the cluster oil swelling, as a result of osmotic processes due to the possible slight and local (a)

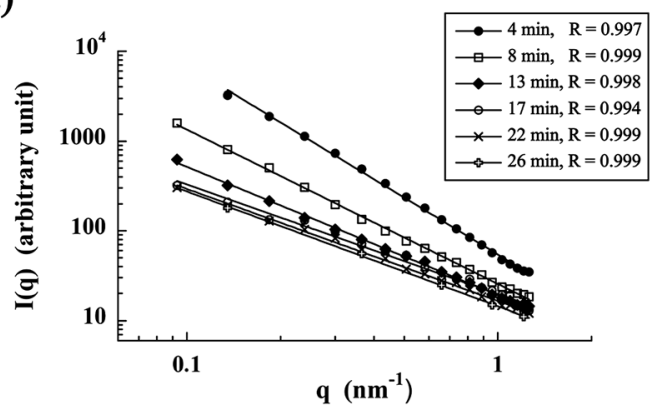

(b)

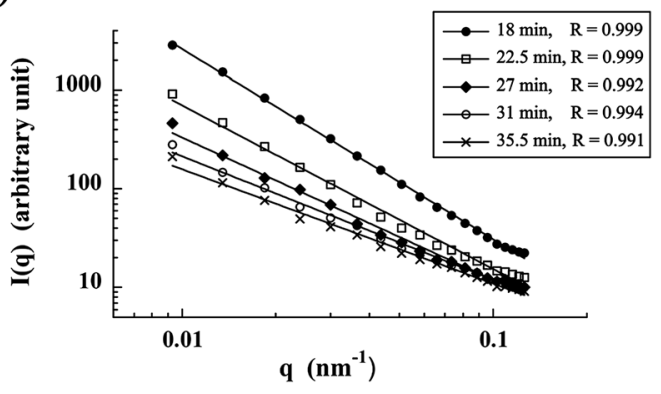

(c)

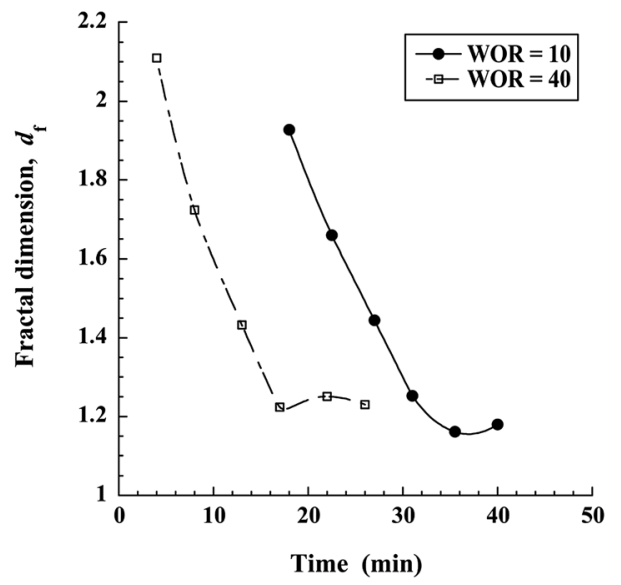

Fig. 5 Static light scattering characterization of the water-in-oil nano-emulsions over time. (a) and (b) present the mean scattered intensity $I(q)$ as a function of the wave vector (i.e. scatter angle), and the evolution of the curves with time. (a) WOR $=10 \%$ and (b) WOR $=40 \%$. (c) Reports the values of fractal dimensions $d_{f}$, obtained from fitting the curves of (a) and (b) with eqn (7), as a function of time.

increase of surfactant concentration in the interstitial spaces within the clusters. The fractal dimension can be accessed all along the aggregation process, and finally stabilized around 1.2, in a very porous state, almost linear chain state (for which $d_{\mathrm{f}}=$ 1 ), even below the values conventionally noted for the DLCA regime for homoaggregation around 1.8. It is to be noted that Fig. 5 reports representative formulations at different WOR values for a constant surfactant concentration (10 wt\%), thus different droplet sizes (see Fig. 3), but showing a similar kinetics of the fractal dimension decrease. This indicates that it is not due to the system properties but due to external factors like the one proposed, driven by cluster oil swelling. As a last remark, the shift of the times for which the aggregation starts between 
these two examples could simply be due to the size of droplets, slightly longer with smaller ones, as expected.

\section{Conclusion}

This study reports for the first time a comprehensive experimental approach that explores and understands the formulation of water-in-oil nano-emulsions by the phase inversion temperature method. The results followed emulsification principles and mechanisms, in the same line as the ones acknowledged for oil-in-water nano-emulsification. Thus, we showed that for temperatures lower than the PIT, the surfactant molecules are concentrated in the aqueous phase. This phase can then be suddenly broken-down by dilution with a fluid oil that penetrates the surfactant network, forming the aqueous nanoemulsion droplets. The study confirms that similar to direct low-energy nano-emulsification with the oil-surfactant ratio, here the water-surfactant ratio has a strong impact on the resulting size of the nano-emulsion droplet. Finally, these reverse nano-emulsions displayed strong adhesive properties, which is common with macro-emulsions but never observed with nano-emulsions, thus leading to the original fabrication of nano-emulsion-gels.

\section{References}

1 M. S. El-Aasser, C. D. Lack, J. W. Vanderhoff and F. M. Fowkes, Colloids Surf., 1986, 29, 103-118.

2 T. Forster, F. Schambil and W. von Rybinski, J. Dispersion Sci. Technol., 1992, 13, 183-193.

3 A. Forgiarini, J. Esquena, C. González and Solans, Langmuir, 2001, 17, 2076-2083.

4 R. Pons, I. Carrera, J. Caelles, J. Rouch and P. Panizza, Adv. Colloid Interface Sci., 2003, 106, 129-146.

5 K. Bouchemal, S. Briançon, E. Perrier and H. Fessi, Int. J. Pharm., 2004, 280, 241-251.

6 J. L. Salager, A. Forgiarini, L. Marquez, A. Pena, M. Pizzino, P. Rodriguez and M. Rondon-Gonzalez, Adv. Colloid Interface Sci., 2004, 108-109, 259-272.

7 T. F. Tadros, P. Izquierdo, J. Esquena and C. Solans, Adv. Colloid Interface Sci., 2004, 108-109, 303-318.

8 C. Solans, P. Izquierdo, J. Nolla, N. Azemar and M. J. GarciaCelma, Curr. Opin. Colloid Interface Sci., 2005, 10, 102-110.

9 N. Anton, P. Gayet, J. P. Benoit and P. Saulnier, Int. J. Pharm., 2007, 344, 44-52.

10 N. Anton, P. Saulnier, A. Béduneau and J. P. Benoit, J. Phys. Chem. B, 2007, 111, 3651-3657.

11 N. Anton, J. P. Benoit and P. Saulnier, J. Controlled Release, 2008, 128, 185-199.

12 N. Anton, J. P. Benoit and P. Saulnier, J. Drug Delivery Sci. Technol., 2008, 18, 95-99.

13 N. Anton, P. Saulnier, C. Gaillard, E. Porcher, S. Vrignaud and J. P. Benoit, Langmuir, 2009, 25, 11413-11419.

14 N. Anton and T. F. Vandamme, Int. J. Pharm., 2009, 377, 142147.

15 T. F. Vandamme and N. Anton, Int. J. Nanomed., 2010, 5, 867873.
16 N. Anton and T. F. Vandamme, Pharm. Res., 2011, 28, 978985.

17 D. J. McClements, Soft Matter, 2012, 8, 1719-1729.

18 F. Ostertag, J. Weiss and D. J. McClements, J. Colloid Interface Sci., 2012, 388, 95-102.

19 A. H. Saberi, Y. Fang and D. J. McClements, J. Colloid Interface Sci., 2013, 391, 95-102.

20 S. Vrignaud, N. Anton, P. Gayet, J. P. Benoit and P. Saulnier, Eur. J. Pharm. Biopharm., 2011, 79, 197-204.

21 F. Hallouard, N. Anton, G. Zuber, P. Choquet, X. Li, Y. Arntz, G. Aubertin, A. Constantinesco and T. F. Vandamme, $R S C$ Adv., 2011, 1, 792-801.

22 A. Klymchenko, E. Roger, N. Anton, H. Anton, I. Shulov, J. Vermot, Y. Mely and T. F. Vandamme, RSC Adv., 2012, 2, 11876-11886.

23 C. Qian, E. A. Decker, H. Xiao and D. J. McClements, Food Chem., 2012, 135, 1440-1447.

24 X. Li, N. Anton, G. Zuber, M. Zhao, N. Messaddeq, F. Hallouard, H. Fessi and T. F. Vandamme, Biomaterials, 2013, 34, 481-491.

25 F. Leal-Calderon, V. Schmitt and J. Bibette, Emulsion science basic principles, Springer, 2007.

26 M. Antonietti and K. Landfester, Prog. Polym. Sci., 2002, 27, 689-757.

27 J. M. Asua, Prog. Polym. Sci., 2002, 27, 1283-1346.

28 E. M. Rosenbauer, K. Landfester and A. Musyanovych, Langmuir, 2009, 25, 12084-12091.

29 S. Vrignaud, N. Anton, C. Passirani, J. P. Benoit and P. Saulnier, Drug Dev. Ind. Pharm., 2013, DOI: 10.3109/ 03639045.2012.730526.

30 M. Porras, C. Solans, C. González, A. Martínez, A. Guinart and J. M. Gutiérrez, Colloids Surf., A, 2004, 249, 115118.

31 N. Usón, M. J. García and C. Solans, Colloids Surf., A, 2004, 250, 415-421.

32 M. Porras, C. Solans, C. González and J. M. Gutiérrez, Colloids Surf., A, 2008, 324, 181-188.

33 L. C. Peng, C. H. Liu, C. C. Kwan and K. F. Huang, Colloids Surf., A, 2010, 370, 136-142.

34 A. H. E. Machado, D. Lundberg, A. J. Ribeiro, F. J. Veiga, B. Lindman, M. G. Miguel and U. Olsson, Langmuir, 2012, 28, 4131-4141.

35 F. Leal-Calderon and P. Poulin, Curr. Opin. Colloid Interface Sci., 1999, 4, 223-230.

36 F. Leal-Calderon, B. Gerhardi, A. Espert, F. Brossard, V. Alard, J. F. Tranchant, T. Stora and J. Bibette, Langmuir, 1996, 12, 872-874.

37 M. von Smoluchowski, Z. Phys. Chem., 1917, 92, 129-168.

38 Kinetic of aggregation and gelation, ed. F. Family and D. P. Landau, Elsevier, Amsterdam, 1984.

39 S. H. Chen, T. L. Lin and J. S. Huang, Physics of complex and supramolecular fluids, Wiley, New York, 1987.

40 P. Meakin, Phase transitions and critical phenomena, Academic Press, New York, 1988, vol. 12.

41 D. A. Weitz and M. Oliveira, Phys. Rev. Lett., 1984, 52, 14331436. 
42 B. B. Mandelbrot, The fractal geometry of nature, Freeman, W. H., San Fransisco, 1982.

43 P. G. J. van Dongen and M. H. Ernst, Phys. Rev. Lett., 1985, 54, 1396-1399.

44 C. M. Sorensen, Aerosol Sci. Technol., 2001, 35, 648-687.

45 P. Sandkühler, J. Sefcik, M. Lattuada, H. Wu and M. Morbidelli, AIChE J., 2003, 49, 1542-1555.

46 P. Sandkühler, M. Lattuada, H. Wu, J. Sefcik and M. Morbidelli, Adv. Colloid Interface Sci., 2005, 113, 65-83.

47 M. L. Broide and R. J. Cohen, Phys. Rev. Lett., 1990, 64, 20262029.

48 D. Ramkrishna, Population balances. Theory and applications to particulate systems in engineering, Academic Press, San Diego, 2000.

49 S. Kumar and D. Ramkrishna, Chem. Eng. Sci., 1996, 51, 1311-1332.

50 P. Sandkühler, J. Sefcik and M. Morbidelli, J. Phys. Chem. B, 2004, 108, 20105-20121.

51 T. A. Witten and L. M. Sander, Phys. Rev. Lett., 1981, 47, 1400-1403.
52 G. Bolle, C. Cametti, P. Codastefano and P. Tartaglia, Phys. Rev. A, 1987, 35, 837-841.

53 J. E. Martin, J. P. Wilcoxon, D. Schaefer and J. Odinek, Phys. Rev. A, 1990, 41, 4379-4391.

54 M. Y. Lin, H. M. Lindsay, D. A. Weitz, R. C. Ball, R. Klein and P. Meakin, Phys. Rev. A, 1990, 41, 2005-2020.

55 D. Asnaghi, M. Carpineti, M. Giglio and M. Sozzi, Phys. Rev. A, 1992, 45, 1018-1023.

56 M. Carpineti and M. Giglio, Adv. Colloid Interface Sci., 1993, 46, 73-90.

57 J. Stankiewicz, M. Cabrerizo-Vilchez and R. Hidalgo-Alvarez, Phys. Rev. E: Stat. Phys., Plasmas, Fluids, Relat. Interdiscip. Top., 1993, 47, 2663-2668.

58 D. Asnaghi, M. Carpinetti, M. Giglio and A. Vailati, Physica A, 1995, 213, 148-158.

59 R. C. Ball, D. A. Weitz, T. A. Witten and F. Leyvraz, Phys. Rev. Lett., 1987, 58, 274-277.

60 A. Béduneau, P. Saulnier, N. Anton, F. Hindré, C. Passirani, H. Rajerison, N. Noiret and J. P. Benoit, Pharm. Res., 2006, 23, 2190-2199. 